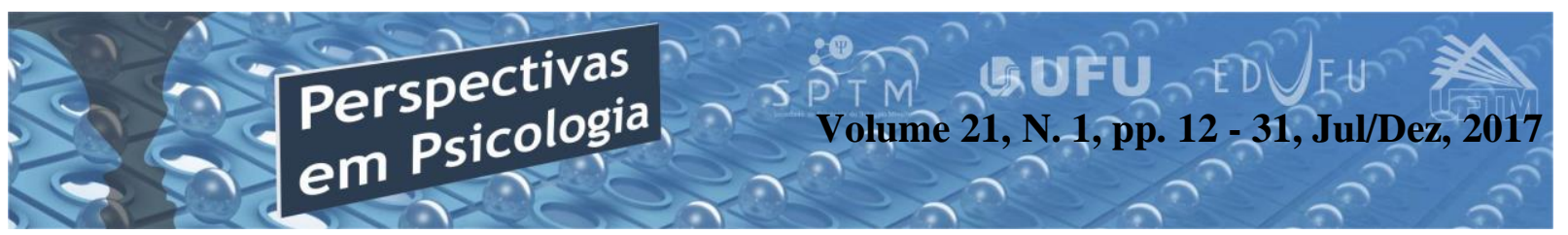

\title{
PERCEPÇÃO DA DOENÇA CARDÍACA E NÍVEIS DE ESTRESSE EM ADULTOS INTERNADOS EM ENFERMARIA
}

\author{
Marineia Crosara de Resende \\ Camila Pereira Teixeira \\ (Universidade Federal de Uberlândia - UFU)
}

\begin{abstract}
Resumo
A doença cardíaca encontra-se entre as mais comuns afecções do mundo moderno e, geralmente, causam grande impacto emocional nos pacientes, incluindo o estresse. Essa pesquisa objetivou caracterizar a percepção do indivíduo sobre sua doença cardíaca; conhecer os níveis de estresse nesses pacientes e correlacionar essas duas variáveis. Participaram 18 pacientes internados em enfermaria de cardiologia, com idade entre 35 e 82 anos. Utilizou-se os instrumentos: ficha de informações sóciodemográficas, Inventário de Percepção da Doença - revisado e o Inventário de Sintomas de Stress para Adultos. Os resultados demonstraram padrões médios na percepção da doença; presença de estresse na maioria dos pacientes, sendo os sintomas psicológicos mais incidentes. Não houve correlação entre as variáveis percepção da doença e níveis de estresse.
\end{abstract}

Palavras-chave: doença cardíaca; percepção da doença; estresse; psicologia.

\section{Abstract \\ Heart Disease Perception and Stress Levels in In-Patients in Ward}

Heart disease is between commoner affections of modern world, and generally it cause great emotional impact on patients, including the stress. The research objective was to characterize the individual heart disease perception, to know the stress levels in these patients and to correlate these two variables. 18 in-patients in the cardiology ward participated, with age between 35 and 82 years. The instruments used were a socio-demographic datasheet, a revised version of the Illness Perception Questionnaire and Lipp's Stress Symptoms Inventory for Adults. The results demonstrated middle standards in illness perception; stress presence in most of patients, with psychological symptoms more incidents. There was no correlation between the variables illness perception and stress levels.

Keywords: heart disease; illness perception; stress; psychology.

\section{Introdução}

"O barulho do coração não é mais

de silêncio, mas de que muita coisa não vai

bem". (paciente S. J. em relato durante atendimento em sua internação). 
As mudanças percebidas no cenário mundial originaram o conceito de transição epidemiológica. Pereira, Alves-Souza e Vale (2015) relatam que essa transição foi conceituada como sendo complexas mudanças nos padrões de saúde e doença e nas interações entre tais padrões e outros fatores consequentes e determinantes, como os demográficos, econômicos e sociais. A Organização Mundial da Saúde (OMS, 2014) define doença crônica como uma doença que tem uma ou mais das seguintes características: ser permanente, produzir incapacidades/deficiências residuais, ser causada por alterações patológicas irreversíveis, obrigar a formação especial do doente para a reabilitação, ou exigir longos períodos de supervisão, observação ou cuidados.

Nesse contexto, estão as Doenças Crônicas Não Transmissíveis (DCNT), consideradas uma ameaça para a saúde e desenvolvimento de todas as nações, isto porque a própria OMS (2014) estima em cerca de 36 milhões as mortes anuais por esse grupo de doenças, cujas taxas de mortalidade já são muito mais elevadas nos países de baixa e média renda. Segundo Malta e Silva Júnior (2013), esse aumento da ocorrência de DCNT é reflexo de alguns efeitos negativos do processo de globalização, da urbanização rápida, da vida sedentária e da alimentação com alto teor calórico, além do consumo do tabaco e do álcool. Esses fatores de risco comportamentais impactam nos principais fatores de risco metabólicos, podendo ocasionar as diversas doenças crônicas existentes. Como exemplos de tais doenças, têm-se as doenças cardiovasculares, a diabetes, a obesidade, as doenças renais, o cancro e as doenças respiratórias (Marques, 2012).

\section{Doença cardíaca}

Diante desse cenário, a doença cardíaca encontra-se entre as mais comuns afecções do mundo moderno, sendo a principal causa de morte no Brasil (IBGE, 2014). De acordo com a última Pesquisa Nacional de Saúde (IBGE, 2014), 4,2\% da população (cerca de 6,1 milhões de pessoas), com 18 anos ou mais tiveram diagnóstico médico de alguma doença do coração no ano de 2013.

As síndromes cardíacas estão entre as razões mais comuns para a ocupação, admissão e readmissão hospitalar (Goodman, Firouzi, Banya, Lau-Walker, \& Cowie, 2012). Entre as nove metas pactuadas para 2015-2025, pela OMS e pelo Plano de DCNT pelo Brasil, destacam-se as metas de redução de $25 \%$ da mortalidade por DCNT, buscando a redução de fatores de risco e tratamento 
dos pacientes de doenças cardiovasculares (Malta, \& Silva Júnior, 2013).

As doenças do coração, geralmente, causam grande impacto psicoemocional sobre os pacientes e, muito especialmente, sobre aqueles que irão se submeter a uma cirurgia cardíaca (Oliveira \& Oliveira, 2010). Para Oliveira e Luz (2010), embora se saiba que fisiologicamente, o comando da vida física e os aspectos comportamentais estejam centralizados no cérebro, é ao "coração" que se atribui ser o centro da vida e que simboliza o sentimento humano. Além disso, um aspecto importante da doença cardíaca, enquanto doença crônica, é que seu início é súbito e, como pode não haver a possibilidade de cura, ela afeta profundamente a vida dos indivíduos, trazendo implicações físicas, sociais e emocionais, além de poder alterar a dinâmica familiar e ser predisponente a alguma incapacidade ao longo do tempo (Marques, 2012).

\section{Doença cardíaca e estresse}

Considerando, então, os aspectos particulares desta enfermidade, citados anteriormente, a doença cardíaca poderá gerar medo, ansiedade e incertezas sobre o que fazer a partir do seu diagnóstico, esses fatores poderão gerar níveis altos de estresse. Para Lipp (2000), o estresse é uma resposta não específica do organismo para lidar com algo que ameaça o seu equilíbrio, quando, por exemplo, o indivíduo se depara com situações extremas que o irritam, amedrontam, excitam, confundem ou que o fazem imensamente feliz (Silva, \& Silva, 2015). O estresse pode ser considerado como um processo, que tem por finalidade adaptar o organismo a uma condição interna ou externa que, de alguma forma, pode estar alterando a percepção de bem-estar que o indivíduo está vivenciando (Silva, \& Silva, 2015).

Segundo o modelo de Lipp (2000), são quatro as fases do estresse: alerta, resistência, quase-exaustão e exaustão. A distinção das mesmas se dá pela duração da ação do estressor e pelo aparecimento de sintomas orgânicos e/ou psicológicos. Nesse sentido, Lipp (2000) define as fases da seguinte forma: a fase de alerta é considerada a fase positiva do estresse; a fase da resistência é aquela em que a pessoa tenta, automaticamente, lidar com os seus estressores com a finalidade de manter seu equilíbrio interno; a fase de quase-exaustão é caracterizada pelo início do processo de adoecimento, em que órgãos mais vulneráveis mostram sinais de deterioração; e, a fase de exaustão é a fase 
final do estresse, em que órgãos mais vulneráveis podem ser acometidos.

A fim de que seja compreendido o estresse, é necessário atentar-se aos eventos estressores que deflagram as reações adaptativas. A doença cardíaca é uma condição que poderá exigir diversos tipos de adaptação, podendo, tornar-se um evento estressor significativo, que pode estar correlacionado com a maneira com que o indivíduo representa e percebe sua doença e encara seu tratamento.

Para Oliveira e Oliveira (2010), muitas condutas intervencionistas nesta doença não são "curativas", mas apenas paliativas e exigem contínua aderência às medidas preventivas, relacionadas ao estilo de vida do paciente, além dos controles médico/laboratoriais regulares. Isto significa que, em alguns casos, mesmo com a intervenção cirúrgica, a doença poderá ser apenas parcialmente tratada, exigindo cuidados permanentes dos pacientes para prevenir ou retardar a progressão da doença, que serão realizados pautados na percepção que eles têm da doença.

Doença cardíaca e percepção da doença

Souza (2014) relata que estudos têm sido desenvolvidos no intuito de investigar a influência da percepção que a pessoa tem sobre sua saúde e sobre a doença, relacionando com aspectos com os quais a pessoa em situação de adoecimento pode enfrentar a patologia. A percepção da doença oferece aos pacientes um panorama dos processos de cuidados de saúde, o que por sua vez determina a forma como o indivíduo vivencia e se adapta à doença (Stafford, Beck, \& Jackson, 2009).

Bonin e outros (2014), em trabalho que visou a construção e a validação de um questionário de conhecimentos sobre sua doença para pacientes com insuficiência cardíaca, pontuam que o conhecimento dos pacientes sobre sua condição é determinante para a aderência e o sucesso de seu tratamento. Em contrapartida, a falta de conhecimento piora a qualidade de vida, produz isolamento social, aumenta as comorbidades, compromete o autocuidado, gera desconhecimento de sinais e sintomas e dificulta a adesão ao tratamento, gerando crenças inadequadas sobre a doença.

As crenças, representações e percepções de doença são designadas na literatura como "cognições de doença", que são crenças implícitas de senso comum que o indivíduo tem sobre sua doença (Marques, 2012). Percepções da doença são crenças e expectativas em relação à sua própria doença e se desenvolvem a partir de uma variedade de fontes, incluindo 
experiência e ambiente (Foxwell, Morley, \& Frizelle, 2013).

É com base nos sistemas de crenças individuais que a informação exterior é interpretada, conferindo uma marca pessoal à forma como o indivíduo percebe e vive a sua saúde e os estados de doença, além do que, essa percepção subjetiva do estado de saúde contribui para a variabilidade de resultados em diferentes esferas, tais como a física e/ou emocional (Malta, 2011; Souza, 2014). Para Goodman e outros, (2012), é reconhecido que a jornada de um paciente com uma doença crônica envolve o desenvolvimento da compreensão de suas necessidades, de seus recursos internos e do apoio externo, de modo que a sua condição possa ser integrada às suas vidas.

Nesse contexto, um dos modelos que surge, com uma visão dinâmica, centrado nas crenças individuais sobre a saúde/doença e nas respostas às ameaças de doença, é o modelo de autorregulação do comportamento de Leventhal e colaboradores. Esse modelo incorpora o modelo de cognições de doença no modelo de autorregulação de comportamento em saúde (Marques, 2012), em que as crenças que a pessoa tem acerca dos sinais e sintomas, bem como a sua interpretação, medeiam as respostas comportamentais perante a ameaça à sua saúde. Esse modelo associa os conceitos teóricos de cognição de doença com as técnicas de resolução de problemas, de modo a explicar o comportamento de coping frente à doença. (Marques, 2012).

O modelo de autorregulação, desenvolvido por Leventhal e outros (Malta, 2011; Marques, 2012), pretendeu compreender a forma como as pessoas entendem o seu estado de saúde, ponderando que a representação da doença e o seu tratamento são influenciados pelas crenças da própria pessoa e da sociedade. Considerando-se, também, que essas crenças podem ser influenciadas por alterações nos padrões comportamentais de saúde e de doença, as experiências vivenciadas, aos diferentes mecanismos de coping adotados, à estrutura psicológica e ao suporte familiar e social, que irão condicionar as percepções da doença. (Malta, 2011).

Os indivíduos podem ter crenças consistentes que podem dar sentido às suas doenças e ajudar a perceber $o$ desenvolvimento dos sintomas (Marques, 2012). As pesquisas dos últimos anos, segundo Marques (2012), configuram-se em investigações acerca das implicações que o diagnóstico, a progressão da doença e as suas condicionantes têm na vida do indivíduo, postulando-se que as implicações físicas, psicológicas e sociais 
parecem ser diferentes, conforme diversos fatores particulares de cada um e da doença em si (Marques, 2012).

No Brasil, ainda são poucos os estudos acerca da percepção do paciente sobre a doença, particularmente no cenário da doença cardíaca (Souza, 2014). Muitos estudos acerca das DCNT e seus múltiplos fatores, que podem estar à elas associados, tem buscado correlacionar estresse e doença cardíaca. Muitos profissionais ainda encaram com certo ceticismo essa associação, encontrando dificuldade em valorizá-la na prática clínica, mesmo sendo o estresse uma das queixas mais comuns em pacientes cardíacos (Bento, Prado, \& Gardenghi, 2015).

Considerando que as doenças do coração, geralmente, causam impacto psicoemocional sobre os pacientes e ponderando-se que esse sofrimento emocional pode potencializar o estresse e comprometer o quadro clínico instalado, essa pesquisa tem os seguintes objetivos:

$\checkmark$ caracterizar a percepção do
indivíduo sobre sua doença
cardíaca;
$\checkmark$ conhecer os níveis de estresse em
adultos cardiopatas;

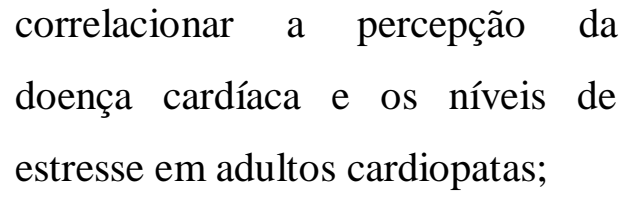
doença cardíaca e os níveis de estresse em adultos cardiopatas;

\section{Método}

\section{Participantes}

Essa pesquisa foi realizada com uma amostra de conveniência retirada do universo de pacientes internados na Enfermaria de Cardiologia do Hospital das Clínicas da Universidade Federal de Uberlândia. (Projeto aprovado pelo Comitê de Ética em Pesquisa da UFU CAAE: 37118214.1.0000.5152). Os critérios de inclusão da pesquisa foram: ser paciente internado na enfermaria de Cardiologia do Hospital de Clínicas da Universidade Federal de Uberlândia, ter 18 anos ou mais, não apresentar comprometimento cognitivo e aceitar participar da pesquisa. Participaram 18 adultos com cardiopatias, com idade entre 35 e 82 anos (média 59,4; $\mathrm{DP}=13,93)$, sendo a maioria homem (61,1\%), casado $(61,1 \%)$, classe socioeconômica média $(72,2 \%)$. As características demográficas podem ser vistas na Tabela 1. 


\section{Tabela 1}

Distribuição de Frequência das Características Sociodemográficas dos Participantes (n=18)

\begin{tabular}{|c|c|c|}
\hline Variável & Porcentagem & Frequência \\
\hline \multicolumn{3}{|l|}{ Sexo } \\
\hline Masculino & 61,1 & 11 \\
\hline Feminino & 38,9 & 7 \\
\hline Total & 100,0 & 18 \\
\hline \multicolumn{3}{|l|}{ Estado Civil } \\
\hline Casado (a)/Convivente & 61,1 & 11 \\
\hline Solteiro $(a)$ & 16,7 & 3 \\
\hline Viúvo $(a)$ & 16,7 & 3 \\
\hline Divorciado (a) & 5,6 & 1 \\
\hline Total & 100,0 & 18 \\
\hline \multicolumn{3}{|l|}{ Classe socioeconomica } \\
\hline Baixa & 22,2 & 4 \\
\hline Média & 72,2 & 13 \\
\hline Alta & 5,6 & 1 \\
\hline Total & 100,0 & 18 \\
\hline \multicolumn{3}{|l|}{ Profissão } \\
\hline Aposentado (a) & 27,76 & 5 \\
\hline Dona de casa & 22,23 & 4 \\
\hline Encarregado de armação & 5,56 & 1 \\
\hline Fazendeiro & 5,56 & 1 \\
\hline Mestre de obras & 5,56 & 1 \\
\hline Operador de máquina & 5,56 & 1 \\
\hline Padeiro & 5,56 & 1 \\
\hline Pedreiro & 5,56 & 1 \\
\hline Produção & 5,56 & 1 \\
\hline Serviços Gerais & 5,56 & 1 \\
\hline Técnico em Eletrotécnica & 5,56 & 1 \\
\hline Total & 100,0 & 18 \\
\hline \multicolumn{3}{|l|}{ Orientação religiosalespiritual } \\
\hline Católica & 44,44 & 8 \\
\hline Evangélica & 44,44 & 8 \\
\hline Outra & 11,11 & 2 \\
\hline Total & 100,0 & 18 \\
\hline \multicolumn{3}{|l|}{ Reside com } \\
\hline Cônjuge & 27,8 & 5 \\
\hline Filhos & 16,7 & 3 \\
\hline Cônjuge e filhos & 16,7 & 3 \\
\hline Cônjuge, filhos e netos & 5,5 & 1 \\
\hline Sozinho & 22,2 & 4 \\
\hline Outros & 11,1 & 2 \\
\hline Total & 100,0 & 18 \\
\hline
\end{tabular}


Com relação ao diagnóstico no momento da internação, $27,8 \%$ apresentam doença arterial coronariana (DAC), 27,8\% infarto agudo do miocárdio, $11,1 \%$ angina instável de alto risco, 11,1\% insuficiência cardíaca, 5,5\% aneurisma de aorta, 5,5\% descolamento de placa na aorta, 5,5\% miocardite lúpica, 5,5\% sopro no coração.

Os pacientes relataram ter descoberto a doença há 2,75 dias em média $(\mathrm{DP}=4,4)$ e estavam internados há 12 dias em média $(\mathrm{DP}=11,3)$.

\section{Instrumentos}

a) Ficha de Informações sócio demográficas- Questionário para coleta de dados sobre idade, gênero, renda mensal, profissão, ocupação, estado civil, tipo de arranjo domiciliar e orientação religiosa/espiritual.

b) Inquérito de Percepção da Doença Revisado (IPQ-R) (Moss-Morris, Weinman, Petrie, Horne, Cameron, Buick, 2002) - Escala que determina a percepção da doença, através da avaliação das crenças relativas à Identidade, às Consequências, à Duração Cíclica, à Duração (aguda/crônica), ao Controle Pessoal, ao Controle pelo Tratamento, à Representação Emocional, à Coerência da doença e às Causas da doença em questão.

A escala encontra-se dividida em três partes, de acordo com Malta (2011): b.1) Componente da Identidade: com lista de 15 sintomas, sobre os quais o participante deve responder "Sim" ou "Não" a duas perguntas: "Desde o diagnóstico da doença cardíaca já sentiu este sintoma?" e "Este sintoma está relacionado com a doença cardíaca?”. Esta escala tem uma variação de 0 a 15 , sendo que a soma das respostas positivas, à última pergunta caracteriza a Identidade da doença.

b.2) Subescalas com as dimensões: a) Duração (aguda/crônica): medição da percepção da duração da doença (contém 6 itens); b)Duração Cíclica: mede a extensão da vulnerabilidade e imprevisibilidade da doença (contém 4 itens); c) Consequências: mede o impacto que a doença tem na vida dos indivíduos (contém 6 itens); d) Controle: mede a percepção que o doente tem sobre o controle da sua doença, e divide-se em duas subdimensões: Controle pelo Tratamento, a qual mede a efetividade do tratamento que é percebida pelo indivíduo (contém 5 itens) e Controle

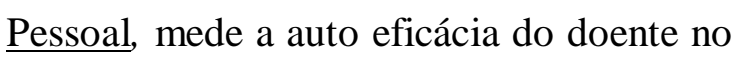
controle da sua doença (contém 6 itens); e) Representação Emocional: mede o estado emocional que está associado à doença (contém 6 itens); f) Coerência da doença: mede até que ponto o indivíduo compreende a sua doença (contém 5 itens). Os itens da escala são apresentados em 
formato likert de (1) discorda plenamente a

(5) concorda plenamente. As dimensões Consequências, Representação Emocional, Duração (aguda/crônica) e Controle Pessoal variam entre 6 e 30, em que os valores mais elevados correspondem respectivamente, a uma representação de maior gravidade das consequências da doença, a uma representação emocional mais negativa, de maior cronicidade e maior controle pessoal. As subescalas Controle pelo Tratamento e Coerência da doença, variam entre 5 e 25 , em que os valores mais elevados correspondem a uma representação de maior eficácia do tratamento e de uma compreensão mais pobre da doença. A dimensão Duração Cíclica, varia entre 4 e 20, em que os valores mais elevados correspondem a uma representação de evolução cíclica da doença.

b.3) Causas: são identificadas 17 causas divididas em a) Atribuições psicológicas: refere-se a causas de origem psicológicas (contém 6 itens); b) Fatores de risco: refere-se a causas relativas a comportamentos/estilo de vida de risco (contém 6 itens); c) Imunidade: refere-se a causas de origem imunológica (contém 3 itens); d) Acaso/Destino, refere-se a causas de origem acidental ou transcendental (contém 2 itens).
Esta parte da escala, também está apresentada sob a forma de escala do tipo Linkert de 5 pontos, a saber, discorda plenamente (1), discorda (2), não discorda nem concorda (3), concorda (4) e concorda plenamente (5). As subdimensões Atribuições psicológicas e Fatores de risco variam entre 6 e 30, a subdimensão Imunidade varia entre 3 e 15 , e a subdimensão Acaso/Destino varia entre 2 e 10.

Após esta classificação, é pedido que o participante enuncie e organize por ordem de importância, as causas mencionadas ou outras, que lhe pareçam ser as causadoras do aparecimento da sua doença.

c) Inventário de Sintomas de Stress para Adultos de Lipp(ISSL - Lipp, 2000) - teste psicológico que visa identificar objetivamente a sintomatologia que o paciente apresenta, avaliando se este possui sintomas de estresse, o tipo de sintoma existente (se somático ou psicológico) e a fase que se encontra.

\section{Procedimentos}

Para a coleta de dados, foi feito contato com os responsáveis pelo setor de Ensino e Pesquisa e da Enfermaria de Cardiologia do HCUFU, solicitando autorização para realização da pesquisa. 
Após aprovação do projeto de pesquisa pelo Comitê de Ética em Pesquisa, foram identificados os pacientes que se enquadram nos critérios de inclusão dessa pesquisa e que estavam internados aguardando procedimentos médicos, tais como exames, cirurgias, dentre outros. Os pacientes anteriormente identificados foram contatados em seus respectivos leitos pelas pesquisadoras e lhes foi informado o motivo da pesquisa, o caráter voluntário da participação e a garantia do sigilo dos dados individuais. Os dados foram coletados no leito em dia e horário previamente agendado e antes do início da aplicação dos instrumentos foi lido e assinado o Termo de Consentimento Livre e Esclarecido (TCLE).

\section{Metodologia para análise dos dados}

Para descrever o perfil da amostra segundo as variáveis em estudo, foram feitas tabelas de frequência das variáveis categóricas, com valores de frequência absoluta (n) e percentual (\%) e estatísticas descritivas das variáveis numéricas, com valores de média, desvio padrão, valores mínimos e máximos, mediana e quartis. Para comparação das variáveis numéricas entre os grupos de níveis de estresse foram utilizados os testes de Mann-Whitney (para duas categorias) e de Kruskal-Wallis (para três ou mais categorias), devido ao tamanho reduzido da amostra e à ausência de distribuição normal dos escores. Para analisar a relação entre as variáveis numéricas foi utilizado o coeficiente de correlação de Spearman. O nível de significância adotado para os testes estatísticos foi de $5 \%$, ou seja, $\mathrm{p}<0,05$.

Em relação aos fatores informados, pelos participantes, que lhes parecem ser as causas da doença cardíaca, estes foram agrupados em três categorias: fatores emocionais (englobando a solidão, preocupação, depressão e estresse); fatores externos (relacionados ao tipo de alimentação, uso de bebida alcoólica e nicotina, sedentarismo) e, os fatores associados à falta de assistência médica, à ação de vírus ou bactéria e à hereditariedade.

\section{Resultados}

Percepção do indivíduo sobre sua doença cardíaca

\section{Dimensão Identidade}

Com relação ao componente da identidade, avaliada pelo relacionamento de sintomas, como, por exemplo, dor, náuseas, falta de ar, fadiga, dor no peito, com a doença cardíaca, os pacientes apresentaram média 7,61 (DP = 2,81), colocam-se levemente acima do ponto 
médio, ou seja, apresentam padrões médios quando à identidade da doença.

Dimensões Duração da doença (aguda/crônica), Duração Cíclica,

Consequências, Controle Pessoal, Controle do Tratamento, Coerência da Doença e

Representação Emocional.

Dimensão Duração da doença

Os pacientes percebem maior cronicidade da doença cardíaca (Média = $21 ; \mathrm{DP}=3,68$ ).

\section{Dimensão Duração cíclica}

Há maior representação da doença relacionada com a vulnerabilidade e a imprevisibilidade da mesma (média 15,78; $\mathrm{DP}=3,02)$.

\section{Dimensão Consequências}

É evidenciada uma representação de maior gravidade da doença com média $24,06(\mathrm{DP}=3,78)$.

Dimensão Representação Emocional

Os dados apontam representação emocional da doença mais negativa (Média $21,06, \mathrm{DP}=5,64)$.

\section{Dimensão Controle}

Em relação à dimensão controle, que se subdivide em Controle Pessoal e Controle do Tratamento, as médias de tais dimensões [Controle Pessoal $(22,78$; DP = 2,88) e Controle do Tratamento (21,39; DP $=2,17)]$ demonstram a percepção dos indivíduos em relação a um controle pessoal importante em relação à sua doença e, a uma crença de maior eficácia no tratamento dispensado à mesma.

\section{Dimensão Coerência da Doença}

Em relação à compreensão da doença, os pacientes apresentaram compreensão mais baixa de sua doença cardíaca (média 14,61; DP = 2,64), colocaram-se levemente abaixo do ponto médio.

\section{Causas}

Quanto à percepção das causas das doenças cardíacas, os pacientes referiramse a: Atribuições Psicológicas (Média 20,61; DP = 4,02), Fatores de risco (Média 21,83; DP $=3,78$ ) e Imunidade (Média 10,44; $\mathrm{DP}=1,89)$. Não acreditam ou desacreditam que seja Acaso/Destino (Média 4,78; DP = 2,05), pois esse item ficou abaixo do ponto médio.

Causas do aparecimento da doença na percepção do paciente

Em relação, em ordem de importância, dos três fatores que lhes parecem ser as causas da doença cardíaca 
estão os fatores emocionais (citados 23 vezes); os fatores externos (9 vezes); e os fatores associados à falta de assistência médica, à ação de vírus ou bactéria e à hereditariedade (foram elencados 11 vezes).

Níveis de estresse em adultos cardiopatas

Entre os participantes dessa pesquisa verificou-se a presença de estresse em $77,8 \%$ dos pacientes (14

Figura 1

Fases de Estresse e Prevalência dos sintomas do Estresse $(n=14)$

\begin{tabular}{|c|c|c|}
\hline & Porcentagem & Frequência \\
\hline Alerta & 7,14 & 1 \\
\hline Resistência & 78,57 & 11 \\
\hline Quase-exaustão & 7,14 & 1 \\
\hline Exaustão & 7,14 & 1 \\
\hline \multirow[t]{2}{*}{ Total } & 100,0 & 14 \\
\hline & Porcentagem & Frequência \\
\hline Sintomas fisicos & 28,6 & 4 \\
\hline Sintomas psicológicos & 64,3 & 9 \\
\hline Ambos os sintomas & 7,1 & 1 \\
\hline Total & 100,0 & 14 \\
\hline
\end{tabular}

Correlações entre percepção da doença cardíaca e os níveis de estresse

Nas Tabelas 2, 3 e 4, Através da correlação de Spearman foi possível verificar que não houve correlação entre pacientes) e ausência em 22,2\% (4 pacientes).

A fase de estresse mais prevalente foi a fase de resistência $(78,57 \%$ ) (ver Figura 1), sendo os sintomas mais incidentes os psicológicos (64,3\%), seguidos por sintomas físicos $(28,6)$ e $\operatorname{ambos}(7,1 \%)$.

4 
Tabela 2

Correlação da Percepção da Doença e a Presença ou Ausência de Estresse

\begin{tabular}{|c|c|c|c|c|c|}
\hline & \multicolumn{2}{|c|}{$\begin{array}{c}\text { Presença de Estresse } \\
(n=14)\end{array}$} & \multicolumn{2}{|c|}{$\begin{array}{c}\begin{array}{c}\text { Ausência de Estresse } \\
(n=4)\end{array} \\
\end{array}$} & \multirow[b]{2}{*}{ Valor $P$} \\
\hline & Média & D.P. & Média & D.P. & \\
\hline Identidade & 8,07 & 2,64 & 6,00 & 3,16 & 0,219 \\
\hline Duração da doença & 21,71 & 3,79 & 18,50 & 1,91 & 0,121 \\
\hline Duração Cíclica & 15,71 & 3,22 & 16,00 & 2,58 & 0,957 \\
\hline Consequências & 24,43 & 3,88 & 22,75 & 3,59 & 0,393 \\
\hline Controle Pessoal & 22,93 & 3,10 & 22,25 & 2,22 & 0,745 \\
\hline Controle Tratamento & 21,21 & 2,26 & 22,00 & 2,00 & 0,592 \\
\hline Coerência da doença & 14,36 & 2,90 & 15,50 & 1,29 & 0,359 \\
\hline Representação emocional & 22,64 & 5,30 & 15,50 & 2,38 & 0,055 \\
\hline Atribuições Psicológicas & 20,14 & 3,82 & 22,25 & 4,86 & 0,364 \\
\hline Fatores de risco & 22,57 & 3,57 & 19,25 & 3,77 & 0,150 \\
\hline Imunidade & 10,07 & 1,77 & 11,75 & 1,89 & 0,085 \\
\hline Acaso/Destino & 4,57 & 1,99 & 5,50 & 2,38 & 0,450 \\
\hline
\end{tabular}

$\mathrm{p} \leq 0,05$

Tabela 3

Correlação da Percepção da Doença e a Fase de Estresse na qual se Encontram

\begin{tabular}{|c|c|c|c|c|c|c|c|}
\hline & \multicolumn{2}{|c|}{$\begin{array}{c}\text { Ausência de Estresse } \\
(n=4)\end{array}$} & \multicolumn{2}{|c|}{$\begin{array}{c}\text { Alerta / Resistência } \\
(n=12)\end{array}$} & \multicolumn{2}{|c|}{$\begin{array}{c}\text { Quase Exaustão / } \\
\text { Exaustão } \\
(n=2)\end{array}$} & \multirow[b]{2}{*}{ Valor $P$} \\
\hline & Média & D.P. & Média & D.P. & Média & D.P. & \\
\hline Identidade & 6,00 & 3,16 & 8,00 & 2,80 & 8,50 & 2,12 & 0,444 \\
\hline Duração da doença & 18,50 & 1,91 & 21,42 & 3,99 & 23,50 & 2,12 & 0,245 \\
\hline Duração Cíclica & 16,00 & 2,58 & 15,42 & 3,40 & 17,50 & 0,71 & 0,637 \\
\hline Consequências & 22,75 & 3,59 & 24,00 & 3,84 & 27,00 & 4,24 & 0,427 \\
\hline Controle Pessoal & 22,25 & 2,22 & 23,25 & 3,25 & 21,00 & 0,00 & 0,366 \\
\hline Controle Tratamento & 22,00 & 2,00 & 20,75 & 2,05 & 24,00 & 1,41 & 0,140 \\
\hline Coerência da doença & 15,50 & 1,29 & 14,58 & 2,81 & 13,00 & 4,24 & 0,585 \\
\hline Representação emocional & 15,50 & 2,38 & 22,42 & 5,73 & 24,00 & 0,00 & 0,149 \\
\hline Atribuições Psicológicas & 22,25 & 4,86 & 20,08 & 4,01 & 20,50 & 3,54 & 0,652 \\
\hline Fatores de risco & 19,25 & 3,77 & 22,25 & 3,72 & 24,50 & 2,12 & 0,242 \\
\hline Imunidade & 11,75 & 1,89 & 10,08 & 1,88 & 10,00 & 1,41 & 0,220 \\
\hline Acaso/Destino & 5,50 & 2,38 & 4,75 & 2,01 & 3,50 & 2,12 & 0,547 \\
\hline
\end{tabular}


Tabela 4

Correlação da Percepção da Doença e os Sintomas de Estresse Apresentados

\begin{tabular}{|c|c|c|c|c|c|c|c|c|c|}
\hline & \multicolumn{2}{|c|}{$\begin{array}{c}\text { Ausência de } \\
\text { Estresse } \\
(n=4)\end{array}$} & \multicolumn{2}{|c|}{$\begin{array}{c}\text { Sintomas Físicos } \\
(n=4)\end{array}$} & \multicolumn{2}{|c|}{$\begin{array}{c}\text { Sintomas } \\
\text { Psicológicos } \\
(n=9)\end{array}$} & \multicolumn{2}{|c|}{$\begin{array}{c}\text { Ambos os } \\
\text { sintomas } \\
(n=1)\end{array}$} & \multirow[b]{2}{*}{$\begin{array}{c}\text { Valor } \\
P\end{array}$} \\
\hline & Média & $D . P$. & Média & D.P. & Média & D.P. & Média & D.P. & \\
\hline Identidade & 6,00 & 3,16 & 6,75 & 2,06 & 8,11 & 2,42 & 13,00 & . & 0,223 \\
\hline Duração da doença & 18,50 & 1,91 & 24,00 & 2,16 & 20,56 & 4,16 & 23,00 & . & 0,181 \\
\hline Duração Cíclica & 16,00 & 2,58 & 13,50 & 3,11 & 16,78 & 3,07 & 15,00 & . & 0,310 \\
\hline Consequências & 22,75 & 3,59 & 23,25 & 4,99 & 24,78 & 3,73 & 26,00 & . & 0,789 \\
\hline Controle Pessoal & 22,25 & 2,22 & 23,25 & 1,50 & 23,33 & 3,39 & 18,00 & . & 0,346 \\
\hline Controle Tratamento & 22,00 & 2,00 & 20,50 & 2,52 & 21,89 & 1,96 & 18,00 & . & 0,268 \\
\hline Coerência da doença & 15,50 & 1,29 & 14,00 & 4,24 & 14,33 & 2,55 & 16,00 & . & 0,584 \\
\hline $\begin{array}{l}\text { Representação } \\
\text { emocional }\end{array}$ & 15,50 & 2,38 & 20,25 & 6,08 & 23,56 & 5,27 & 24,00 & . & 0,184 \\
\hline Atribuições Psicológicas & 22,25 & 4,86 & 22,25 & 3,86 & 19,11 & 3,82 & 21,00 & . & 0,501 \\
\hline Fatores de risco & 19,25 & 3,77 & 24,00 & 2,94 & 21,89 & 3,98 & 23,00 & . & 0,397 \\
\hline Imunidade & 11,75 & 1,89 & 9,00 & 2,58 & 10,44 & 1,33 & 11,00 & . & 0,269 \\
\hline Acaso/Destino & 5,50 & 2,38 & 5,75 & 2,63 & 3,89 & 1,54 & 6,00 & . & 0,370 \\
\hline
\end{tabular}

\section{Discussão}

O presente estudo apontou alguns resultados interessantes ao considerar o contexto em questão. De maneira geral, os resultados mostraram que apesar da grande prevalência de estresse na referida população, este não teve significativa correlação com a maneira pela qual os pacientes percebem sua doença cardíaca.

De acordo com os resultados, com relação à percepção que os participantes dessa pesquisa têm de sua doença cardíaca, quanto ao componente da identidade, não há uma identificação completa de estarem doentes, nem de estarem sãos. Foxwell e outros (2013) chamam atenção para a importância de entender como, verdadeiramente, os pacientes compreendem sua cardiopatia, o que pode influenciar na maneira total do sistema de crenças e de emoções vivenciadas a partir dessa compreensão. Muitos pacientes coronarianos têm equívocos sobre sua doença, sendo fundamental compreender o impacto do diagnóstico, das interpretações equivocadas e dos sentimentos e sintomas 
que essa desencadeia (Stafford e outros, 2009; Foxwell e outros, 2013).

Os pacientes dessa pesquisa percebem maior cronicidade da doença cardíaca, ou seja, compreendem que a doença traz vulnerabilidade e que é imprevisível e grave. Os dados também apontam para uma representação emocional mais negativa da doença. $\mathrm{O}$ coração tem sido compreendido como sendo o centro da vida, mesmo que essa relação gere discussão, se pensada em termos apenas fisiológicos (Oliveira, \& Luz, 2010). Essa condição traz percepções que geram emoções negativas, fazendo acreditar na gravidade e vulnerabilidade diante dessa doença. Para Souza e David (2010), o sistema cardiovascular recebe grande influência dos estados emocionais, ou seja, a forma como cada pessoa vivencia uma situação, seja ela boa ou ruim, irá interferir intensamente no funcionamento cardíaco, e nesse sentido pode-se compreender a grande ligação entre a percepção de aspectos emocionais junto ao cenário das doenças cardíacas.

Os indivíduos participantes acreditam no controle pessoal de sua doença e na eficácia do tratamento. Embora tenham referido à baixa compreensão de sua doença cardíaca. Ribeiro et al. (2014) apresentaram um estudo brasileiro no qual o conhecimento dos sintomas dos pacientes é inadequado e poderia ser um fator de risco significativo, para fatores como adesão ao tratamento, autocuidado e vivência da condição clínica. A partir da compreensão correta do diagnóstico, bem como de sua cronicidade e cuidados a serem dispensados, pode-se melhorar o humor, os aspectos emocionais e outros fatores envolvidos no processo de tratamento (Goodman e outros, 2012). Em relação a alta crença sobre o autocontrole da doença, Goodman e outros, (2012) relatam que pacientes com maiores crenças se co-responsabilizam em seu processo de tratamento, e que quanto melhor a compreensão da doença, maior adesão aos cuidados que necessitam ser tomados.

Quanto à percepção das causas das doenças cardíacas, os pacientes referiramse à atribuições psicológicas, fatores de risco e imunidade. Não acreditam ou desacreditam que seja acaso ou destino. Como causas principais, os pacientes perceberam estar relacionadas aos fatores emocionais, aos fatores externos e aos fatores associados à falta de assistência médica, à ação de vírus ou bactéria e à hereditariedade.

Bento e outros (2015) colocam que a importância de avaliar o nível de estresse durante o período de internação em pacientes cardiopatas se deve justamente pelas alterações fisiológicas, 
principalmente no sistema cardiovascular, que são desencadeadas pelo estresse. E, é a partir dessa investigação que se pode focar a eliminação da fonte de estresse e garantir melhor recuperação para o doente cardíaco internado. Os resultados mostraram a presença de estresse na maioria dos pacientes, sendo a fase de resistência a mais prevalente, com os sintomas psicológicos sendo os incidentes. Esse estresse pode ser advindo, do grande impacto emocional em receber $\mathrm{o}$ diagnóstico de uma cardiopatia. Souza e David (2010) afirmam que estudos nessa área pontuam que o estresse emocional estimula vias serotonérgicas no sistema nervoso central que irão interferir diretamente no sistema cardiovascular, e esse sistema participa ativamente das adaptações ao estresse (Bento e outros, 2015).

Embora nessa pesquisa não tenha sido encontrada correlações entre a percepção da doença cardíaca e o estresse, em estudo realizado por Wolever e outros (2015), averiguou-se que 20\% dos pacientes que haviam sofrido infarto agudo do miocárdio apresentaram altos níveis de estresse quando comparado com outras populações médicas. Os autores colocam ainda que entre $60 \%$ a $90 \%$ das consultas médicas tem relacionamento com estresse e, este com impactos em vários sistemas, inclusive o cardiovascular.

Mesmo não tendo sido encontradas correlações nessa pesquisa, entre as variáveis do estudo, é importante considerar a necessidade de atenção aos pacientes com cardiopatias, já que as causas emocionais foram as principais mencionadas e encontradas em níveis consideráveis.

Estudos realizados em outros países encontraram forte associação entre a percepção da doença cardíaca e fatores emocionais, entretanto, estes se referiam à ansiedade e depressão (Stafford e outros, 2009; Goodman e outros, 2012; Mussatto e outros, 2013).

\section{Considerações Finais}

Esse estudo pôde evidenciar a percepção dos pacientes sobre sua doença, verificar a alta incidência de estresse, com prevalência da fase de resistência e predomínio dos sintomas psicológicos, não tendo sido evidenciado correlação entre a percepção da doença cardíaca e os níveis de estresse apresentado pelos participantes. É preciso considerar que a amostra foi reduzida, o que indica a necessidade de outros estudos para análises mais profundas.

Novas pesquisas poderiam focar a 
relação da percepção da doença cardíaca com outras variáveis psicológicas, tais como depressão e ansiedade. O estudo da percepção da doença é uma área de pesquisa pertinente para doenças do coração, justamente por elencar significativas correlações com os fatores emocionais que necessitam ser analisados, investigados e tratados (Stafford e outros, 2009; Foxwell e outros, 2013).

Foxwell e outros (2013), por meio de uma revisão sistemática da literatura acerca do tema "relação entre percepção da doença, humor e qualidade de vida em população com problemas cardíacos", discorrem que estudos sugerem que a percepção da doença é um importante determinante ao longo do curso da doença cardíaca, com impactos no humor, depressão e ansiedade. Fatores esses que podem levar ao estresse, que tem alta prevalência em pacientes diagnosticados com doença cardíaca.

Wolever e outros (2015) afirmam que problemas psicológicos, como estresse afetam vários sistemas do corpo, podendo desenvolver, dentre outras doenças, a doença cardíaca. Nesse sentido, entender os aspectos psicológicos relacionados à doença cardíaca, como é a percepção e as crenças dos pacientes em relação às doenças, auxiliará a pensar em intervenções psicológicas, que irão contribuir no prognóstico e até mesmo na prevenção de doenças e, particularmente, no ajustamento psicológico frente a doença e à vida.

\section{Referências}

Bento, D. C. P., Prado, A. P., \& Gardenghi, G. (2015). Estudo dos níveis de estresse dos pacientes submetidos à angioplastia durante a internação na unidade de terapia intensiva. Revista Eletrônica Saúde e Ciência, 2 (1), 7-21. Recuperado em 26 de Agosto de 2015 de: http://www.rescceafi.com.br/vol2/n1/Artigo-I-7-21.pdf

Bonin, C. D. B., Santos, R. Z. dos, Ghisi, G. L. M., Vieira, A. M., Amboni, R., \&Bentti, M. (2014). Construção e validação do Questionário de Conhecimentos para pacientes com Insuficiência Cardíaca. Arquivos Brasileiros de Cardiologia, 102 (4), 364 - 373. http://dx.doi.org/10.5935/abc.20140032. 
Foxwell, R., Morley, C., \& Frizelle, D. (2013). Illness perception, mood and quality of life: A systematic review of coronary heart disease patients. Journal of Psychosomatic Research, 75, 211-222. http://dx.doi.org/10.1016/j.jpsychores.2013.05.003

Goodman, H., Firouzi, A., Banya, W., Lau-Walker, M., \& Cowie, M. R. (2012). Illness perception, self-care behaviour and quality of life of heart failure patients: A longitudinal questionnaire survey. International Journey of Nursing Studies , 50, 945-953. http://dx.doi.org/10.1016/j.ijnurstu.2012.11.007

Instituto Brasileiro de Geografia e Estatística (2014). Pesquisa Nacional de Saúde 2013 Percepção do estado de saúde, estilos de vida e doenças crônicas. IBGE, Rio de Janeiro. Recuperado em 03 de Setembro de 2014 de: ftp://ftp.ibge.gov.br/PNS/2013/pns2013.pdf Lipp, M. E. N. (2000). Manual do Inventário de Sintomas de Stress para Adultos de Lipp (ISSL). São Paulo: Casa do Psicólogo.

Malta J. I. R. F. (2011) Diabetes: Percepção da doença e auto cuidado. XII Curso de Mestrado em Saúde Pública. Portugal: Escola Nacional de Saúde Pública, Lisboa. Recuperado em 01 Agosto de 2014 de: http://hdl.handle.net/10362/9403

Malta, D. C., \& Silva Júnior, J. B. da. (2013). O Plano de Ações Estratégicas para o Enfrentamento das Doenças Crônicas Não Transmissíveis no Brasil e a definição das metas globais para o enfrentamento dessas doenças até 2025: uma revisão. Epidemiologia e Serviços de Saúde, 22 (1), 151-164. http://dx.doi.org/10.5123/S167949742013000100016

Marques, L. J. L. (2012). Percepção do doente insuficiente renal crónico a efectuar tratamento de substituição renal. Dissertação. Portugal: Escola Superior de Saúde de Viseu, Viseu. Recuperado em 26 de Agosto de 2015 de: http://hdl.handle.net/10400.19/1761

Moss-Morris, R., Weinman, J., Petrie, K., Horne, R., Cameron, L., \&Buick, D. (2002) The revised illness perception questionnaire (IPQ-R). Em Psychology\& Health, 17 (1), 1-16. Recuperado em 15 de Agosto de 2015 de: http://www.uib.no/ipq/pdf/The\%20revised\%20illness\%20perception\%20questionnaire.pd $\underline{f}$

Mussatto, K. A., Sawin, K.J., Schiffman, R., Leske, J., Simpson, P., \& Marino, B. S. (2013).The importance of self-perceptions to psychosocial adjustment in adolescents with heart disease. Journal Pediatric Health Care, 28 (3), 251-261. http://dx.doi.org/10.1016/j.pedhc.2013.05.006 
Oliveira, M.F. P. de, \& Luz,P. L. da. O impacto da cirurgia cardíaca. (2010). Em Melo-Filho, J. de. Psicossomática Hoje. 343-349. Porto Alegre: Artmed.

Oliveira, S. A., \& Oliveira, M. F. P. Psicologia e Cardiologia: um desafio que deu certo. (2010) Em Ribeiro, A. L. A. \&Gagliani, M. L. (orgs). Psicologia e Cardiologia: um desafio que deu certo. 1-6. São Paulo: Atheneu.

Pereira, R. A., Alves-Souza, R. A. \& Vale, J. S. (2015). O processo de transição epidemiológica no Brasil: uma revisão. Revista Científica da Faculdade de Educação e Meio Ambiente, v. 6, n.1, pp. 99-108. Acessado em: 23 de agosto de 2015. Recuperado em 30 de Agosto de 2015 de: http://www.faema.edu.br/revistas/index.php/RevistaFAEMA/article/view/322

Ribeiro, V., Melão, F., Rodrigues, J. D., Leite, S. M., Garcia, R. M., Dias, P. \& Maciel, M. J. (2014). Perception of illness symptoms in patients with acute coronary syndrome: A need to improve. Revista Portuguesa de Cardiologia, v. 33, n. 9, pp. 519-523. http://dx.doi.org/10.1016/j.repc.2013.09.017

Silva, D. P. da.\& Silva, M. N. R. M. O. (2015). O Trabalhador com estresse e intervenções para o cuidado em saúde. Trabalho, Educação e Saúde, 13 (1), 201214.https://dx.doi.org/10.1590/1981-7746-sip00032

Souza, F. S. O. de, \& David, J. H. Quando o coração falha: marca-passo e cardioversor desfibrilador cardíaco. (2010). Em Ribeiro, A. L. A. \&Gagliani, M. L. (orgs). Psicologia e Cardiologia: um desafio que deu certo. 57-64. São Paulo: Atheneu.

Souza, S. P. A. (2014). Percepção da doença e adesão ao tratamento em um grupo de pacientes com hipotireoidismo. Dissertação. São Cristóvão: Universidade Federal de Sergipe. Recuperado em 15 de Agosto de 2015 de: http://bdtd.ufs.br/tde_busca/arquivo.php?codArquivo=1593

Stafford, L., Berk, M. \& Jackson, H. J. (2009). Are illness perceptions about coronary artery disease predictive of depression and quality of life outcomes? Journal of Psychosomatic Research, 66, 211-220. http://dx.doi.org/10.1016/j.psychores.2008.09.005

Wolever, R. Q., Goel, N. S., Roberts, R. S., Caldwell, K., Kliger, B., Dusek, J. A., Perlman, A., Dolor, R. \& Abrams, D.I. (2015). Integrative Medicine patients have high stress, pain, and psychological symptoms. Explore, $11 \quad$ (4), 296-303. http://dx.doi.org/10.1016/j.explore.2015.04.003

Organização Mundial da Saúde (OMS, 2014). Global status report on noncommunicable diseases 2014.Switzerland: World Health Organization. Recuperado em 20 de Agosto de 
2015 de: http://www.who.int/.

\section{As Autoras:}

Marineia Crosara de Resende é Psicóloga, Professora do Instituto de Psicologia, Tutora/orientadora da Psicologia no Programa de Residência Multiprofissional em Saúde pela Universidade Federal de Uberlândia. Universidade Federal de Uberlândia, Instituto de Psicologia. Av. Pará, 1720, Campus Umuarama, CEP 38405382 - Uberlândia, MG - Brasil, Telefone: (34) 3218-2185

Camila Pereira Teixeira é Psicóloga, Residente Multiprofissional em Saúde pela Universidade Federal de Uberlândia.

Recebido em: 27/04/2017.

Aprovado em: 19/06/2017. 